\title{
Hábitos de vida e condições de saúde dos caminhoneiros do Brasil: uma revisão da literatura
}

\author{
Habits of life and health of truck drivers in brazil: a literature review
}

\author{
Angélica Alessia, Márcia Keller Alves ${ }^{b}$ \\ a Acadêmica do Curso de Bacharelado em Nutrição da Faculdade Cenecista de Bento Gonçalves, RS.

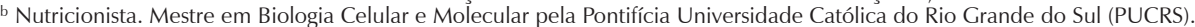 \\ Docente da Faculdade Cenecista de Bento Gonçalves.
}

Introdução: O trabalho como motorista de caminhão pode levar a prejuízos à saúde, pois favorece a adoção de estilos de vida pouco saudáveis, além de práticas que deixam estes profissionais vulneráveis a doenças. Sedentarismo, hábitos alimentares inadequados, excesso de peso e nível de estresse elevado são fatores que contribuem para o aparecimento das mesmas.

Objetivo: Revisar os hábitos de vida e condições de saúde dos caminhoneiros do Brasil.

Materiais e Métodos: Foi realizado levantamento bibliográfico na Base de Dados MedLine através do site de busca da Biblioteca Virtual em Saúde, usando as palavras-chaves "caminhoneiro", "caminhão" e "motorista de caminhão". Para a seleção, foi aplicado o filtro "ano de publicação", buscando apenas artigos publicados a partir de 2002. Os artigos encontrados foram selecionados quanto à originalidade e relevância, considerando-se a adequação do delineamento, número amostral e relação do conteúdo com as questões de pesquisa.

Resultados: Foram identificados 61 artigos e incluídos 18 trabalhos que apontam fatores de risco para doenças crônicas não transmissíveis, bem como a presença destas doenças em alguns caminhoneiros. Verificou-se ainda que a trajetória do caminhoneiro interfere em sua saúde, pois o torna vulnerável a adoção de hábitos de vida pouco saudáveis, comportamento que pode ser encontrado em profissionais submetidos a condições semelhantes de trabalho.

Conclusão: Os caminhoneiros possuem hábitos de vida nocivos à saúde, tais como alimentação não saudável, falta de atividade física, tempo insuficiente de descanso, vícios (cigarro e álcool), além do uso de drogas, exposição a doenças transmissíveis e ausência de controle periódico em saúde. Isto pode estar relacionado às doenças encontradas nestes profissionais, como hipertensão arterial, excesso de peso, diabetes mellitus, dislipidemias e doenças infectocontagiosas.

Palavras-chave: fatores de risco; estilo de vida; riscos ocupacionais; saúde do trabalhador.

Introduction: Truck driving is an activity that can lead to health issues due to the unhealthy lifestyle inherent to the job demands and also due to practices which expose them to diseases. Sedentary lifestyle, inadequate habits, overweight and stressful activities are factors that contribute to disease development in this group of people.

Objective: To review the life and health habits of truck drivers in Brazil.

Materials and Methods: It was conducted a bibliographic database (MEDLINE) search through the Virtual Health Library site, using the keywords "truck", "truck" and "truck driver". The filter "year of publication" was applied, seeking only articles published since 2002. Articles were selected for originality and relevance, considering the adequacy of the design, sample size and link between its content and the research questions.

Results: We identified 61 and included 18 studies that pointed to risk factors for chronic diseases, and the presence of these diseases in some truck drivers. It was also found that the trajectory of the truck driver interferes with their health because it makes them vulnerable to the adoption of unhealthy lifestyle habits, behavior that can be found in professionals subjected to similar conditions. Conclusion: The truck drivers have harmful lifestyle habits to health, such as unhealthy diet, physical inactivity, insufficient rest time, addictions (cigarettes and alcohol), and the use drugs, exposure to communicable diseases and the absence of periodic health control. These may be related to the diseases found in these professionals, such as high blood pressure, excess weight, diabetes mellitus, dyslipidemia and infectious diseases.

Keywords: risk factors; life style; occupational risks; occupational health 


\section{INTRODUÇÃO}

A saúde é o maior recurso para o desenvolvimento social, econômico e pessoal, assim como uma importante dimensão da qualidade de vida. Fatores políticos, econômicos, sociais, culturais, ambientais, comportamentais e biológicos podem tanto favorecer como prejudicar a saúde 1 . O Brasil tem experimentado mudanças no perfil epidemiológico da população, com alterações relevantes no quadro de morbimortalidade. As doenças infectocontagiosas, que representavam cerca de metade das mortes registradas no país em meados do século XX, hoje são responsáveis por menos de $10 \%$, ocorrendo o oposto em relação às doenças cardiovasculares ${ }^{2}$.

As profundas mudanças observadas, nas últimas décadas, no padrão etário da mortalidade, por sexo, bem como na estrutura das causas da mortalidade da população brasileira, são devidas não só ao aumento das causas cardiovasculares, respiratórias e neoplasias, como também estão relacionadas, fortemente, ao aumento da mortalidade por causas externas, com efeitos inevitáveis sobre o processo de envelhecimento da população, uma vez que atingem, prioritariamente, contingentes do sexo masculino nas faixas etárias muito jovens e jovens-adultos, em todo o território nacional ${ }^{2}$. O monitoramento da prevalência dos fatores de risco para doenças, principalmente os de natureza comportamental (dieta, sedentarismo, dependência química - de tabaco, álcool e outras drogas), é uma das ações mais importantes da vigilância; sobre essas evidências observadas, podemse implementar ações preventivas de maior poder custoefetivo ${ }^{3}$.

Entre vários aspectos socioeconômicos, a escolaridade tem sido apontada como importante determinante da situação de saúde de um indivíduo. A baixa escolaridade está presente em diversos estudos realizados com motoristas de caminhão. Masson e Monteiro ${ }^{4}$ mostraram em seu estudo média inferior a 8 anos de estudo em $64,8 \%$ dos caminhoneiros, tendo como médias regionais 5,4 anos para o Nordeste, 7,5 anos para o Sudeste e 7,2 anos para a região Sul do Brasil. Resende et al. ${ }^{5}$ mostraram que apenas $2 \%$ dos motoristas apresentaram relação com o ensino superior, e que $67 \%$ dos motoristas estudaram até o ensino fundamental. Dos entrevistados somente $22 \%$ completaram o ensino médio.

O trabalho do motorista de caminhão pode levar a prejuízos à saúde, pois acaba favorecendo a adoção de estilos de vida pouco saudáveis como sedentarismo, inadequados hábitos alimentares, sobrepeso, uso de álcool e tabaco, entre outros. A profissão também interfere na convivência familiar e vida social do motorista ${ }^{4}$. Assim, o objetivo deste trabalho foi revisar os hábitos de vida e condições de saúde dos caminhoneiros do Brasil.

\section{MATERIAIS E MÉTODOS}

O presente estudo trata-se de uma revisão de literatura referente os hábitos de vida e condições de saúde dos caminhoneiros do Brasil. Para a construção desta revisão, foi operacionalizado o percurso metodológico por meio das etapas descritas a seguir. A primeira etapa constituiu-se na formulação da questão da pesquisa: quais são os hábitos de vida e as condições de saúde dos caminhoneiros do Brasil? Diante deste questionamento, partiu-se para a segunda etapa cujo propósito foi o de selecionar as publicações que constituíram a amostra.

Para identificar os estudos publicados acerca dos hábitos de vida e condições de saúde dos caminhoneiros foi utilizada a busca online à Base de Dados MedLine através do site de busca da Biblioteca Virtual em Saúde (BIREME), usando as palavras-chaves "caminhoneiro", "caminhão" e "motorista de caminhão". Os critérios para a seleção da amostra foram: que o estudo abordasse no título a temática investigada; foi aplicado o filtro "ano de publicação", buscando apenas artigos publicados a partir de 2002; apresentasse o texto na íntegra e cujas pesquisas tenham sido realizadas no Brasil, com o público-alvo em questão e publicados em português nos idiomas português, inglês e espanhol.

Os artigos encontrados foram selecionados quanto à originalidade e relevância, considerando-se a adequação do delineamento, número amostral e relação do conteúdo com as questões de pesquisa. Os dados utilizados neste estudo foram devidamente referenciados, respeitando e identificando seus autores e demais fontes de pesquisa, observando rigor ético quanto à propriedade intelectual dos textos científicos pesquisados.

Os dados foram analisados segundo Gil ${ }^{6}$ através de quatro leituras: 1) exploratória (na qual os resultados obtidos através da busca na base de dados foram analisados de acordo com os objetivos da pesquisa); 2) seletiva (na qual foram selecionados apenas aqueles que de fato interessam à pesquisa); 3) analítica (que teve como finalidade ordenar e sumariar as informações contidas nas fontes, possibilitando a obtenção de respostas às questões de pesquisa); e 4) interpretativa (na qual foi-se relacionando o que o autor afirmava com as questões propostas neste estudo).

\section{RESULTADOS E DISCUSSÃO}

As buscas resultaram em um total de 61 artigos no site da BIREME, sendo excluídos 13 inicialmente através do filtro "ano de publicação". A partir da leitura seletiva dos 48 artigos restantes, foram incluídos 18 artigos que se adequarem à 
questão da pesquisa do presente estudo. A leitura analítica e interpretativa foi realizada nos 18 artigos, relacionando o seu conteúdo com as questões de pesquisa.

Os resultados foram organizados em duas categorias. A categoria 1 foi denominada "Hábitos de Vida dos Caminhoneiros"; e a categoria 2, "Doenças Encontradas nos Caminhoneiros". Dentro destas categorias, foram incluídos tópicos de temas relacionados.

\section{HÁBITOS DE VIDA DOS CAMINHONEIROS}

\subsection{Alimentação}

Os resultados do estudo de Lago $^{7}$ apontam elevada frequência de fatores de risco cardiovasculares nos motoristas de caminhão, o que pode ser reflexo de má alimentação, falta de condicionamento físico e nível de estresse elevado.

Em geral, os caminhoneiros de rota longa se alimentam em restaurantes, à beira das estradas, com oferta de alimentos de alto valor calórico e baixo valor nutritivo, assim como calorias vazias, vindas especialmente através do uso de álcool. Má digestão e sensação desagradável no estômago são sintomas comuns aos caminhoneiros ${ }^{8}$.

No estudo de Guedes et al. ${ }^{9}$, foi observado que $26,8 \%$ dos entrevistados relataram acrescentar sal à alimentação depois de preparada. Quanto aos fatores de risco para hipertensão arterial, os autores declaram que não se deve associar a doença somente à hereditariedade, pois os hábitos de vida - tal como a alimentação - estão mais ligados diretamente a este fator, e contribuem para o surgimento da hipertensão arterial ou o seu agravamento. Além disso, a ingestão de álcool está diretamente relacionada ao desenvolvimento de hipertensão arterial.

Resende et al. ${ }^{5}$ mostraram que a alimentação dos motoristas de caminhão é precária. A grande maioria dos motoristas prepara a comida em local adaptado como cozinha, na parte externa do caminhão. Notou-se que a refeição é simples e sem variedade.

Penteado et al. ${ }^{10}$ mostra que $33,25 \%$ dos caminhoneiros entrevistados disseram ter uma alimentação gordurosa sempre e 51,25\% relataram ter uma alimentação gordurosa eventualmente.

\subsection{Sedentarismo}

Masson e Monteiro ${ }^{4}$ relataram que $77,1 \%$ dos caminhoneiros estudados são sedentários. Do mesmo modo, Lago ${ }^{7}$ classificou $100 \%$ dos caminhoneiros entrevistados como sedentários, enquanto que Cavagioni et al. ${ }^{8}$ apontaram que $74 \%$ dos entrevistados foram classificados como sedentários.

Coser et al. ${ }^{11}$ mostraram que foi relatado pelos participantes a dificuldade da prática regular e contínua de exercícios, devido aos horários do trabalho, à duração das viagens e ao tempo disponível, o que dificulta esta prática saudável por parte destes profissionais.

\subsection{Horas de descanso}

Os motoristas de caminhões não dormem o suficiente, e, nesta profissão um descanso adequado é extremamente necessário para que o caminhoneiro desempenhe sua função da melhor maneira possível, sendo que qualquer tipo de comprometimento pode causar graves acidentes.

De acordo com o estudo de Resende et al. ${ }^{5}$, a preocupação com o sono se faz necessária, pois $16 \%$ dos participantes da pesquisa que têm viagens entre 1501 e 3000 $\mathrm{km}$ faz parte do grupo que raramente ou nunca dorme de quatro a oito horas por dia. Estão também nesse grupo $22 \%$ dos entrevistados, os quais geralmente percorrem distâncias de 801 a 1501 km. Por fim, o número é ainda maior (25\%) para os que percorrem mais de $3000 \mathrm{~km}$.

Mais da metade dos caminhoneiros estudados por Wendler et al. ${ }^{12}$ relatou dormir entre 3 e 5 horas por dia, e muitos, sob ação dos psicoestimulantes, passam mais de 2 dias sem dormir. Entre os caminhoneiros estudados por Nascimento et al. ${ }^{13}$, a quantidade de horas de sono por dia encontrada foi de 4 a 6 horas por dia. Este estudo mostrou significância estatística entre as variáveis horas de descanso e acidentes nas estradas com o uso de álcool e uma correlação considerada moderada, o que sugere que menos horas de descanso ou sono podem contribuir moderadamente no envolvimento em acidentes sob o efeito do álcool.

Moreira e Gadani ${ }^{14}$ apresentaram resultados preocupantes: a maioria dos caminhoneiros (51\%) dorme entre 3 e 6 horas por dia, enquanto uma outra parcela (22\%) dorme entre 1 a 3 horas por noite, apenas. Masson e Monteiro 4 relatam que os entrevistados dormiam em média de 5,3 horas na semana em que estavam trabalhando e 7,9 horas no final de semana.

Em um cenário composto por dormir no caminhão, ter uma qualidade de sono ótima e uma alimentação ótima ou regular, $11 \%$ dos entrevistados por Resende et al. ${ }^{5}$ encaixamse no grupo dos que nunca ou raramente têm esse tipo de situação. Quando se muda a variável qualidade do sono de ótima para boa, o índice de caminhoneiros que compõem o grupo dos que raramente ou nunca têm essa rotina chega a $22 \%$. Se adicionarmos nesse grupo os caminhoneiros que nunca ou raramente têm boa qualidade do sono dormindo no caminhão e uma alimentação regular, esse número chega a $33 \%$ dos entrevistados.

Leal ${ }^{15}$ aponta que o local de descanso são os postos de gasolina, nos quais $87 \%$ dos caminhoneiros entrevistados dizem parar para dormir. Longas distâncias e prazos curtos podem ser os fatores determinantes para que esses 
profissionais não durmam adequadamente e tenham prejuízos físicos e psicológicos ${ }^{5}$.

\subsection{Tabagismo}

Em relação aos aspectos de saúde e estilo de vida estudados por Masson e Monteiro ${ }^{4}$ foi observado que $21 \%$ dos motoristas eram tabagistas; eles consumiam em média de 17 cigarros ao dia.

Guedes et al. ${ }^{9}$ mostraram o uso de tabaco por $23,7 \%$ dos entrevistados, enquanto que Coser et al. ${ }^{11}$ encontraram uma prevalência de $20 \%$ de fumantes no grupo de caminhoneiros estudados. Penteado et al. ${ }^{10}$ encontraram prevalência de $21,25 \%$ de usuários regulares ("sempre") e 11,25\% de usuários irregulares ("às vezes").

\subsection{Uso de álcool}

Nascimento et al. ${ }^{13}$ observaram que $91 \%$ dos caminhoneiros fazia uso de bebidas alcoólicas nas jornadas de trabalho, dos quais $24 \%$ utilizava o álcool todos os dias e $35 \%$ o consumia de duas a três vezes por semana. Este trabalho mostrou que o local onde o álcool é consumido pode influenciar moderadamente o envolvimento do caminhoneiro em acidentes nas estradas: dos 14 caminhoneiros que haviam se envolvido em acidentes, 11 deles (79\%) faziam uso de álcool em postos de combustíveis.

Moreira e Gadani ${ }^{14}$ mostraram que $69 \%$ dos motoristas de caminhão não usam álcool durante o horário de trabalho. Dos 31\% que afirmaram usar, disseram utilizar juntamente com o rebite para o efeito ser intensificado nas primeiras horas ou enquanto esperam a carga e carregamento, à noite e à tarde.

Guedes et al. ${ }^{9}$ mostraram que o consumo de bebidas alcoólicas foi relatado por $49,4 \%$ dos caminhoneiros pesquisados. O estudo de Penteado et al. ${ }^{10}$ dividiu os motoristas que usam sempre álcool (4\%) daqueles que usam às vezes $(39,5 \%)$. O consumo de álcool entre os entrevistados por Villarinho et al. ${ }^{16}$ é maior (84\%), seguido de maconha (33\%). O uso de álcool pode contribuir para o envolvimento do caminhoneiro em situações de risco, tal como o acesso de trabalhadores do sexo, que se aproveitam da situação ${ }^{16}$.

Embora a legislação proíba a venda de bebidas alcoólicas e a comercialização de anfetaminas em postos de combustíveis, há a necessidade de campanhas preventivas e educativas voltadas para esta categoria profissional, além de fiscalização criteriosa nas estradas a respeito de dirigir sob efeitos de álcool e outras drogas. Os caminhoneiros devem ser alertados sobre os riscos de dependência de substâncias (como álcool e drogas psicoativas ou "rebites"), em um trabalho intensivo de informação e conscientização visando à melhoria da qualidade de vida desses profissionais.

\subsection{Uso de substâncias psicoativas - "rebites"}

Entre os caminhoneiros, o uso de substâncias para reduzir o sono e diminuir o cansaço é uma realidade: $46 \%$ da amostra estudada por Nascimento ${ }^{17} \mathrm{faz}$ uso deste tipo droga, conhecida por "rebite". O uso, segundo o mesmo autor, está relacionado a três fatores principais: motivos econômicos (dirigir por mais tempo, percorrer longos percursos sem muitas paradas), pressão da empresa (especialmente quando a carga é perecível) e dependência.

Nascimento et al. ${ }^{13}$ verificaram que $66 \%$ dos caminhoneiros de estrada costumavam fazer uso de anfetaminas durante o percurso de viagem, dentre os quais $27 \%$ as usavam diariamente e $60 \%$ de duas a três vezes por semana. As anfetaminas eram adquiridas nos postos de combustíveis. Estes dados são semelhantes aos encontrados por Moreira e Gadani $^{14}$, que encontraram o uso em $65 \%$ dos entrevistados. Os caminhoneiros relataram que usam rebites com principal objetivo de chegar ao local estabelecido no dia e hora marcada, onde os princípios ativos mais utilizados foram femproporex e anfepramona (antigamente denominada dietilpropiona), ambos derivados da anfetamina.

Em relação ao uso de medicamentos, Masson e Monteiro ${ }^{4}$ mostraram que a maioria dos entrevistados $(54,2 \%)$ fazia uso de drogas psicoativas para se manterem acordados, devido à necessidade de percorrerem longas distâncias e sofrerem pressão do tempo para a entrega de mercadorias. Entre estes motoristas, 54,4\% ingeriam até cinco comprimidos de anfetamina por viagem e 43,8\% usavam a droga há mais de 10 anos.

Segundo mostra o estudo de Villarinho et al. ${ }^{16}$, é comum o uso do rebite nos motoristas (17\%), além de outras drogas em menor escala: cocaína (12\%), "bolinha" (estimulantes, geralmente anfetaminas - 3\%), calmantes $(5 \%)$, cola $(4 \%), \operatorname{LSD}(3 \%)$ e crack $(1,5 \%)$. O consumo frequente de medicamentos para inibir o sono foi relatado por $49,4 \%$ dos entrevistados por Guedes et al. ${ }^{9}$, sendo que $33,1 \%$ relataram dirigir cansados e $30 \%$ relataram que algumas vezes perdem a atenção.

Wendler et al. ${ }^{12}$ mostraram que $96,88 \%$ dos entrevistados afirmaram ter feito uso pelo menos uma vez ou utilizar rotineiramente medicamentos com a finalidade de aumentar o tempo de vigília. A compra ocorre em postos de gasolina, borracharia, farmácia, restaurantes, lanchonete, mercadinho e no Paraguai.

Moreira e Gadani ${ }^{14}$ verificaram que o uso de rebite é injustificável, uma vez que, na sua amostra, 65\%, transportam cargas não perecíveis (grãos), ou seja, sem risco de perda. Ainda assim, esses trabalhadores se defendem e afirmam 
que mesmo não transportando cargas perecíveis, também cumprem horário.

Segundo Moreira e Gadani ${ }^{14}$, o principal motivo de acidentes letais de caminhoneiros nas estradas está relacionado com o fato de dormirem ao volante, já que se vêm obrigados a fazer uso de substâncias (cafeína e rebite, principalmente) para entregar as cargas no prazo estabelecido.

Wendler et al. ${ }^{12}$ afirmaram vários relatos de motoristas que, sob a ação das anfetaminas, param seus caminhões no meio da pista ou invadem a pista contrária em razão das alucinações causadas. O estudo de Nascimento et al. ${ }^{13}$ mostrou que o percentual de caminhoneiros envolvidos em acidentes nas estradas devido ao uso de anfetaminas e álcool é de $27 \%$ e $17 \%$, respectivamente, sendo os postos de combustíveis os locais para compra e/ou consumo destas substâncias.

Wendler et al. ${ }^{12}$ mostraram em seu estudo que os efeitos colaterais do uso de rebite mais comuns apontados pela sua amostra foram: insônia, midríase, visão turva, agitação, boca seca, irritabilidade, ansiedade, tensão nervosa, cefaleia, sudorese, alucinação e palpitação. Efeitos colaterais como insônia e visão turva aumentam o risco de acidentes nas estradas, já que esses efeitos aparecem quando estão dirigindo ${ }^{13}$.

É preciso uma fiscalização mais intensa por parte da Agência Nacional de Vigilância Sanitária (ANVISA) em postos e locais de circulação de caminhoneiros, a fim de impedir a comercialização ilegal e clandestina, assim como o uso indiscriminado de substâncias psicoativas. Além disso, é preciso reavaliação dos prazos de entrega por parte dos proprietários dos caminhões, de modo a reduzir as chances de uso de anfetaminas durante o período de trabalho.

\subsection{Ausência de controle periódico em saúde}

Para Masson e Monteiro ${ }^{4}$, os caminhoneiros estudados deixaram a saúde em "segundo plano", pois grande parte dos participantes da pesquisa referiu não ir ao médico há muito tempo, não utilizavam os serviços de saúde quando estão viajando e não terem tempo para cuidar da saúde. Além disso, verificou-se que o estresse é hoje um dos principais problemas entre os caminhoneiros.

\subsection{Ausência de medidas preventivas contra Doenças Sexualmente Transmissíveis}

O nível de escolaridade dos caminhoneiros estudados por Rocha ${ }^{18}$ foi baixo, uma vez que a maioria não possuía sequer o ensino fundamental completo. Este estudo mostrou que quanto maior a escolaridade maior adesão ao uso de preservativo, e, portanto, maior adesão às medidas de prevenção sobre o HIV e doenças sexualmente transmissíveis (DST). Teles et al. ${ }^{19}$ mostraram que quase um terço dos caminhoneiros estudados possuía no máximo 4 anos de escolaridade. O relato de DST neste estudo mostrou-se estatisticamente associado à escolaridade.

A escolaridade dos caminhoneiros estudados por Leal ${ }^{15}$ é relativamente baixa, uma vez que $68,8 \%$ dos entrevistados tem até o ensino fundamental completo. Percebeu-se neste estudo relação inversa entre a faixa etária e o nível de escolaridade, e que, com relação à escolaridade e o conhecimento sobre as formas de transmissão do HIV (foco do estudo), aqueles caminhoneiros com menor escolaridade têm menos informações sobre o assunto.

No estudo de Villarinho et al. ${ }^{16}$ são resumidas as práticas que deixam os caminhoneiros vulneráveis à infecção por HIV e outras DST. Dentre elas, destacam-se as relações extraconjugais, a banalização do risco por aqueles que estão casados e o fato de não usar preservativo em todos os tipos de relações sexuais (vaginal, anal e/ou oral). As razões mais citadas para a não-prevenção foram: ficar sem ter relação sexual, resistir à prática do fazer sexo sem camisinha, diminuir o número de parceiras e selecionar com quem transar.

Nascimento ${ }^{17}$ conclui em seu trabalho que os comportamentos observados entre os caminhoneiros estão fundamentados em princípios socialmente constituídos com base na cultura machista, levando a práticas que tornam os caminhoneiros vulneráveis à DST e AIDS.

Rocha ${ }^{18}$ mostrou que o comportamento sexual dos caminhoneiros é de risco, uma vez que a maioria procura por sexo quando fica mais do que 20 dias longe de casa e $8,8 \%$ não usa preservativo. Dos caminhoneiros estudados, $26,7 \%$ já tiveram algum tipo de DST, sendo a gonorreia a doença mais comum entre eles.

Para $4 \%$ dos caminhoneiros estudados por Villarinho et al. ${ }^{16}$, o preservativo não diminui o risco de contrair HIV. O uso de preservativo nestes sujeitos é diferenciado segundo tipo de parceira e prática: com as parceiras fixas houve uso mínimo do preservativo (6,0\%); com as parceiras frequentes houve uso de preservativo na relação vaginal $(56,6 \%)$ e anal $(45,0 \%)$, porém houve poucos que usavam preservativo quando faziam sexo oral $(6,3 \%)$; com as parceiras casuais, o uso de preservativo foi frequente na relação vaginal (67\%), oral (46\%) ou na anal $(54 \%)$. Isto mostra que a parceira fixa é a mais vulnerável no que se refere à infecção pelo HIV ou qualquer outra doença sexualmente transmissível.

Nascimento ${ }^{17}$ mostra em seu estudo que tanto caminhoneiros casados quanto solteiros relatam contatos sexuais com parceiras variadas, indicando certa tendência entres estes caminhoneiros para a busca de mulheres diversificadas. Os principais motivos para a busca de contato sexual na 
estrada foram solidão, problemas familiares, machismo, aventura e falta de vergonha.

Verifica-se, deste modo, que a exposição ao risco de infecção por HIV e outras doenças sexualmente transmissíveis se estende às esposas ou parceiras fixas dos caminhoneiros, uma vez que todos os estudos supracitados mostram que eles não usam preservativo em suas relações sexuais em casa, com suas parceiras. Nascimento ${ }^{17}$ apresenta como principais motivos para a não-prevenção a relações de confiança (com a parceira), fragilidade do preservativo e incômodo; enquanto que os motivos para o uso de preservativo são anticoncepção e desconfiança do cônjuge.

A carência de assistência à saúde e de prevenção junto aos motoristas é grande, de modo que Leal ${ }^{15}$ mostra que $3 \%$ dos caminhoneiros citaram que a intervenção relativa à HIV/AIDS ou saúde ali vivenciada, foi a única já sofrida por eles. Apenas 10,4\% do total de entrevistados relataram ter sofrido abordagem em campanhas de Organizações Não Governamentais (ONG), e muitos acham ineficiente apenas a distribuição de material gráfico (folhetos) e veem como positivas as campanhas que envolvam palestras, esclarecimento de dúvidas e conversas.

Propostas de prevenção (HIV e AIDS) em âmbito nacional, respeitadas as peculiaridades regionais, que possam dar apoio a caminhoneiros, são de extrema necessidade. A prevenção nos locais de trabalho dos caminhoneiros parece ser promissora, pois permite entender melhor seu universo. Deve-se, ainda, envolver a esposa/cônjuge dos caminhoneiros nas campanhas para uso de preservativo, a fim de que elas não desconfiem da proposta do uso de camisinha pelo parceiro.

\section{DOENÇAS DETECTADAS NOS CAMINHONEIROS}

\subsection{Hipertensão arterial}

Cavagioni et al. ${ }^{8}$ mostraram que $20 \%$ dos motoristas de caminhão estudados apresentaram pressão arterial com valores compatíveis com hipertensão arterial, mas desconheciam ser hipertensos e dentre aqueles que referiram fazer uso de anti-hipertensivos a grande maioria não estava com a pressão arterial controlada ( $<140 / 90 \mathrm{mmHg}$ ).

Guedes et al. ${ }^{9}$ mostraram que $18,7 \%$ dos caminhoneiros estudados relataram possuir hipertensão arterial, sendo que $33,4 \%$ desses não faziam o tratamento medicamentoso. No entanto, ao realizar a avaliação, observou-se que 20,7\% possuíam níveis pressóricos característicos para o quadro de hipertensão arterial.

Através da medida da pressão arterial, Lago ${ }^{7}$ constatou que $39,3 \%$ dos entrevistados apresentaram a pressão arterial elevada, enquanto que Coser et al. ${ }^{11}$ encontraram a prevalência de hipertensão arterial sistólica e diastólica em $47 \%$ da sua amostra.

\subsection{Sobrepeso e obesidade}

Domingos et al. ${ }^{20}$ observaram em seu estudo sobrepeso em $44,6 \%$ dos caminhoneiros, obesidade em $27,2 \%$ e apenas $28,2 \%$ dos motoristas com índices de massa corporal (IMC) considerados adequados. No entanto, se forem somados apenas os indivíduos que estão acima do peso, chega-se a $71,8 \%$ da amostra, que representa a maioria. O mesmo foi encontrado por Lago ${ }^{7}$ : o estado nutricional da maioria dos motoristas de caminhão estudados, obtido através do cálculo do IMC, foi classificado em sobrepeso $(35,7 \%)$ e obesidade (50,0\%), ou seja, $85,7 \%$ estão acima do peso considerado normal.

A média de peso apresentada pelos motoristas estudados por Masson e Monteiro ${ }^{4}$ foi de $81 \mathrm{~kg}$ e a maioria apresentava sobrepeso, ou seja, IMC entre 25 a $29,9 \mathrm{~kg} / \mathrm{m}^{2}$ (40,9\%), com média de IMC de $27,5 \mathrm{~kg} / \mathrm{m}^{2}$.

A amostra de Coser et al. ${ }^{11}$ constituiu-se de $42 \%$ dos caminhoneiros com obesidade, $44 \%$ com sobrepeso e $78 \%$ com obesidade visceral.

\subsection{Diabetes Mellitus}

Os exames bioquímicos mostraram incidência de hiperglicemia (possível diabetes) em 42,8\% dos indivíduos entrevistados por Lago ${ }^{7}$. Coser et al. ${ }^{11}$ não encontraram nenhum caso de Diabetes mellitus, porém alguns indivíduos apresentaram intolerância à glicose (valores de glicose entre 100 e $125 \mathrm{mg} / \mathrm{dL})$.

\subsection{Dislipidemias}

Coser et al. ${ }^{11}$, em seu estudo, mostrou através da análise do perfil lipídico da população estudada que 45\% dos indivíduos apresentaram valores de colesterol total aumentados e $42 \%$ possuem elevação da lipoproteína de baixa densidade (Low Density Lipoprotein - LDL). Além disso, 29\% dos participantes do estudo demonstram níveis elevados de triglicerídeos. A classificação de dislipidemias revelou o predomínio de hipertrigliceridemia isolada, que pode ser explicado pelos hábitos alimentares inadequados e a falta de atividade física por parte dos caminhoneiros.

\subsection{Doenças infectocontagiosas: HIV/AIDS e hepatite B}

A AIDS vem sendo tratada como uma doença com caráter de cronicidade, que acarreta a necessidade de avaliação e acompanhamento, a fim de subsidiar continuamente a estruturação dos serviços para atender a novas demandas que 
possam surgir no contexto de vida de pessoas soropositivas, visando à integralidade e à melhor qualidade da atenção em saúde ${ }^{21}$

Em relação à hepatite, os vírus $\mathrm{B}, \mathrm{C}$ e D são aqueles que têm a possibilidade de causar doença crônica. Eventualmente, o diagnóstico é realizado quando aparecem sinais e sintomas em face das complicações da doença, porém há indivíduos com infecção crônica que não apresentam manifestações clínicas e são considerados portadores assintomáticos. Contudo, são capazes de transmitir hepatite e têm importância epidemiológica na perpetuação da endemia ${ }^{22}$.

Berra et al. ${ }^{23}$ encontraram uma prevalência alta $(0,9 \%)$ de caminhoneiros soropositivos e cerca de três vezes este índice se fossem considerados os indivíduos pertencentes à faixa etária de 41 a 55 anos, nos quais foram detectadas 1,76\%, amostras positivas.

Teles et al. ${ }^{19}$ mostraram que $35,6 \%$ dos caminhoneiros estudados afirmaram ter sido ou ser portador de DST, e que esta condição estava associada à idade (maior de 30 anos), à escolaridade (menos de 8 anos de estudos), ao tempo de profissão (superior a 10 anos), a relações com profissional do sexo, entre outros fatores. Com relação à história passada de DST, 22,5\% dos entrevistados por Villarinho et al. ${ }^{16}$ referiram já ter tido pelo menos um episódio; destes, 54\% afirmaram ter contraído essas doenças em relações com profissionais do sexo, $40 \%$ com amigas, e $5 \%$, com moças que pegam na estrada.

O estudo de Teixeira e Sesti ${ }^{24}$ revelou a prevalência de positividade de 1,1\% para o antígeno de superfície HBsAg e 14,3\% para o anti-HBc. Ao comparar a soro positividade e fatores de risco associados ao $\mathrm{HBV}$, foi constado que o resultado positivo para o HBsAg e Anti-HBc é devido ao fato de não fazer uso de preservativos nas relações sexuais (69\%), não ter parceira fixa $(15,3 \%)$ e não tomar a vacina.

Os caminhoneiros são um grupo eminentemente do gênero masculino, itinerante, e diversos estudos mostram que estão vulneráveis às DST/AIDS, uma vez que, assim como as prostitutas, são descritos como "população ponte" em função de suas múltiplas parcerias sexuais ${ }^{14}$. Conhecer estas vulnerabilidades pode auxiliar na definição de políticas de prevenção e autocuidado, considerando o contexto vivido e as experiências individuais de sexualidade.

O caminhoneiro é um profissional que está mais vulnerável a doenças como as DST e HIV/AIDS. A vulnerabilidade do caminhoneiro, no entanto, não está somente associada ao tempo que permanece fora de casa, mas à cultura inerente a essa categoria. Foi possível perceber que motoristas permanecem acordados por longas horas, o que, somado a hábitos alimentares inadequados e sobrepeso, termina por influenciar o desempenho dos motoristas ao volante, diminui a capacidade de reação e, consequentemente, aumenta o risco de acidentes nas rodovias brasileiras.

\section{CONSIDERAÇÕES FINAIS}

Esta revisão apresentou uma análise dos hábitos de vida e condições de saúde dos caminhoneiros do Brasil. Os estudos apontam que os caminhoneiros possuem hábitos de vida nocivos à saúde, tais como alimentação não saudável, falta de atividade física, tempo insuficiente de descanso, vícios (cigarro e álcool), além do uso de drogas, exposição a doenças transmissíveis e da ausência de controle periódico em saúde. Estes hábitos podem estar relacionados às doenças encontradas nestes profissionais, como hipertensão arterial, excesso de peso, diabetes mellitus, dislipidemias, além das doenças infectocontagiosas. Além disso, as ações em saúde para este público são raras e isoladas. Assim, recomenda-se fortemente a elaboração de propostas regulares de promoção da saúde dos caminhoneiros e de atividades que visem ao diagnóstico e à prevenção de doenças em âmbito nacional, de maneira correta e universal, respeitadas as peculiaridades regionais.

\section{REFERÊNCIAS}

1. Ministério da Saúde (BR). Secretaria de Políticas de Saúde. Projeto Promoção da Saúde. As cartas da promoção da saúde. Brasília: Ministério da Saúde; 2002.

2. Instituto Brasileiro de Geografia e Estatística. Indicadores sócio demográficos e de saúde no Brasil. Rio de Janeiro: IBGE; 2009.

3. Malta DC, Cesário AC, Moura L, Neto OLM, Silva J, Jarbas B. A construção da vigilância e prevenção das doenças crônicas não transmissíveis no contexto do Sistema Único de Saúde. Epidemiol Serv Saúde. 2006;15(3):47-65. http://dx.doi.org/10.5123/s167949742006000300006

4. Masson VA, Monteiro MI. Estilo de vida, aspectos de saúde e trabalho de motoristas de caminhão. Rev Bras Enferm. 2010;36(4):533-40. http://dx.doi.org/10.1590/S0034-71672010000400006

5. Resende PTV, Souza PR, Cerqueira PR. Hábitos de vida e segurança dos caminhoneiros brasileiros. In: Simpósio de Administração da Produção, Logística e Operações Internacionais. Anais do SIMPOI 2010; 2010 ago. São Paulo: FGV; 2010. p. 1-17.

6. Gil AC. Como delinear uma pesquisa bibliográfica. In: Gil AC. Como elaborar projetos de pesquisa. São Paulo: Atlas; 2002. p. 59-85.

7. Lago M. Perfil lipídico e fatores de risco cardiovasculares em motoristas de caminhão da região norte do estado do Rio Grande do Sul [Monografia]. Carazinho: Universidade Luterana do Brasil; 2009.

8. Cavagioni LC, Pierin AMG, Batista KM, Bianchi ERF, Costa ALS. Agravos à saúde, hipertensão arterial e predisposição ao estresse em motoristas de caminhão. Rev Esc Enferm USP. 2009;43(Esp 2): 1267-71.

9. Guedes HM, Brum KA, Costa PA, Almeida MEF. Fatores de risco para o desenvolvimento de hipertensão arterial entre motoristas caminhoneiros. Cogitare Enferm. 2010 out./dez.;15(4):652-8. 
10. Penteado RZ, Gonçalves CGO, Costa DD, Marques JM. Trabalho e saúde em motoristas de caminhão no interior de São Paulo. Saúde Soc. 2008;17(4):35-45. http://dx.doi.org/10.1590/S010412902008000400005

11. Coser J, Fontoura S, Fontoura T, Rizzi C. Triagem dos perfis lipídico e glicídico em caminhoneiros que trafegam no centro unificado de fronteira, entre Brasil e Argentina. RBAC. 2009;41(3):223-8.

12. Wendler EA, Busato CR, Miyoshi E. Uso de anfetaminas por motoristas de caminhão para reduzir o sono. Publ UEPG Ci Biol Saude. 2003;9:7-14.

13. Nascimento EC, Nascimento E, Silva JP. Uso de álcool e anfetaminas entre caminhoneiros de estrada. Rev Saude Publica. 2007;41(2):290-3.

14. Moreira RS, Gadani JAAB. A prevalência do uso de anfetaminas por caminhoneiros que passam pela cidade de Dourador-MS. Interbio, 2009;3(2):27-34.

15. Leal AF. No peito e na raça: a construção da vulnerabilidade de caminhoneiros - um estudo antropológico de políticas públicas para HIV/AIDS no Sul do Brasil [tese]. Porto Alegre: Universidade Federal do Rio Grande do Sul; 2008.

16. Villarinho L, Bezerra I, Lacerda R, Latorre MRDO, Paiva V, Stall R, Hearst N. Caminhoneiros de rota curta e sua vulnerabilidade ao HIV, Santos, SP. Rev Saúde Pública. 2002;36(4):61-7. http://dx.doi. org/10.1590/S0034-89102002000500009

17. Nascimento E. Desenvolvimento de pesquisa-ação com caminhoneiros de estrada: trabalhando na problematização as questões voltadas à sexualidade, DST/AIDS e drogas [tese]. Ribeirão Preto: Universidade de São Paulo; 2003.
18. Rocha EM. DST e AIDS em regiões de fronteiras: um estudo com caminhoneiros no estado de Rondônia [dissertação]. Brasília: UnB; 2008.

19. Teles AS, Matos MA, Caetano KAA, Costa LA, França DDS, Pessoni GC, Brunini SM, Martins RMB. Comportamentos de risco para doenças sexualmente transmissíveis em caminhoneiros no Brasil. Rev Panam Salud Publica. 2008;24(1):25-30. http://dx.doi. org/10.1590/S1020-49892008000700003

20. Domingos JBC, Jora NP, Carvalho AMP, Pillon SC. Consumo de álcool, sobrepeso e obesidade entre caminhoneiros. Rev Enferm UERJ. 2010;18(3):377-82.

21. Seidl EMF, Zannon CMLC, Tróccoli BT. Pessoas vivendo com HIV/ AIDS; enfrentamento, suporte social e qualidade de vida. Psicol Reflex Crit. 2005;18(2):188-95. http://dx.doi.org/10.1590/S010279722005000200006

22. Ministério da Saúde (BR). Secretaria de Vigilância à Saúde. Secretaria de Atenção à Saúde. Diretrizes e recomendações para o cuidado integral de doenças crônicas não transmissíveis: promoção da saúde, vigilância, prevenção e assistência. Brasília: Ministério da Saúde; 2008.

23. Berra JAP, Bacetti L, Alves K, Fiório V. Soroprevalência de HIV em caminhoneiros usuários da Rodovia Anhanguera, SP 330, Brasil. Rev Inst Adolfo Lutz. 2003;62(3):171-6.

24. Teixeira CC, Sesti LFC. Soroprevalência de HBsAg e Anti-HBc em caminhoneiros da região central do Rio Grande do Sul. News Lab. 2011;107:184-90. 